\title{
The Treatment of Women with Post-Menopausal Undifferentiated Arthralgia -The First Report of Efficacy of Hormone Replacement Therapy
}

\author{
Kiyomitsu Miyachi' ${ }^{1,2 *}$, Belinda Sasse ${ }^{1,3}$, So Nomoto ${ }^{4}$, Toshiko Igarashi ${ }^{2}$, Shinichi Mashiba ${ }^{2}$ and \\ Koyama Takao ${ }^{5}$ \\ ${ }^{1}$ Rheumatology, women's health and aging, Japan \\ ${ }^{2}$ First Diagnostic Division, Health Sciences Research Institute Inc, Japan \\ ${ }^{3}$ Department of Rheumatology, Monash Health, Australia \\ ${ }^{4}$ Department of Orthopedics, Saiseikai Yokohama Tobu Hospital, Japan \\ ${ }^{5}$ Obstetoric and Gynecology, Koyama Takao Clinic, Japan
}

*Corresponding author: Kiyomitsu Miyachi, First Diagnostic Division, Health Sciences Research Institute Inc, 2-2 Ichibanishinakacho Tsurumi-ku Yokohama, Kanagawa, 230-0023, Japan

To Cite This Article: Kiyomitsu M, Belinda S, So Nomoto, Toshiko I, Shinichi M, Koyama T. The Treatment of Women with Post-Menopausal Undifferentiated Arthralgia -The First Report of Efficacy of Hormone Replacement Therapy. Am J Biomed Sci \& Res. 2019 - 1(3). AJBSR. MS.ID.000525. DOI: 10.34297/AJBSR.2019.01.000525

Received: February 06, 2019 | Published: February 15, 2019

\begin{abstract}
Objective: Peri- and post-menopausal women commonly present with morning stiffness and joint pain, often without joint swelling or pathological abnormalities, presenting clinicians with diagnostic and management troubles. This study evaluates the use of Hormone Replacement Therapy (HRT) for symptomatic relief in these patients to discriminate early stage of rheumatoid arthritis.

Methods: 174 female patients were screened between January and September in 2018. Rheumatoid factor (RF), anti-CCP antibody, CRP, estradiol (E2), and follicle stimulating hormone (FSH) testing was performed; 24 with morning stiffness but no significant joint pain, 27 patients meeting criteria for defined autoimmune rheumatic disease, or 16 with declined treatment and 24 with miscellaneous diseases were excluded. Of the 83 remaining UA,15 were perimenopausal and prescribed Tocopherol N, and 68 were postmenopausal and prescribed HRT. Patients were reassessed following two to six months of treatment. Women who achieved a $30 \%$ reduction or greater in p-VAS scores with treatment were considered to have responded.
\end{abstract}

Results: $12 / 15$ (76\%) perimenopausal, and 57/68 (83.3\%) postmenopausal women met response criteria. resulting from the E2 levels higher/ FSH levels lower and p-VAS, reduction, compared to baseline $\mathrm{P}<0.001$.

Conclusion: HRT likely provides effective symptomatic relief in patients with postmenopausal undifferentiated arthritis. It may also provide relief in perimenopausal women, but sample sizes were small in these groups.

Keywords: Undifferentiated arthritis (UA); Premenopausal arthralgia (PrMA); Postmenopausal arthralgia (PoMA); Estrogen deficiency; Rheumatoid arthritis (RA); Hormone replacement therapy (HRT)

\section{Introduction}

It is well known morning stiffness and arthralgia commonly affect peri- and postmenopausal women, often associated with mild swelling of the peripheral and distal interphalangeal joints (PIPs, DIPs), mimicking early rheumatoid arthritis (RA) or osteoarthritis $(\mathrm{OA})[1,2]$. What happened at peri-and post-menopausal stage? Estradiol levels will be fluctuated and depleted at these stages, respectively, in contrast to men. After menopause, estradiol level of women is going down to a third of level of men [3]. Lack of estradiol induces autoimmunity through activation of NFkB [4,5]. This arthralgia consists primarily of pain on motion and tenderness; a diagnosis of undifferentiated arthritis based on a score of less than 5 by the ACR/EULAR criteria for the diagnosis of early RA is usually made [6]. Recently, it was reported that almost half RA diagnosis are misdiagnosed and proper diagnosis in most cases were OA or menopausal arthralgia suspected [7]. 
In this study, we presented typical two cases responded to hormone replacement therapy, although they were initially diagnosed as an early stage of RA and autoimmune rheumatic disease.

Case1 was born in 1960. Her menopause was noted at a 46-year-old in 2004. She noticed numbness on peripheral joints and could not sleep well in 2008. She visited my clinic because of peripheral joint pains and elbow joint pain. Her blood test showed CRP 0.03, RF 50U, anti-CCP Ab 9.2U, E2 less than 11.8 and FSH 88.4 in May 2014 and ANA 160 homogenous, which was proved as antiDFS Ab positive (50.5 Index) later.

HRT started immediately and her joint pain was reduced to $39 \%$ at one month later, and $7 \%$ at 10 months later. However, she newly complained of left wrist joint pains in August in 2018 and X ray showed bone erosion associated with radiocarpal joint space narrow and received $8 \mathrm{mg}$ weekly. Her pain was gradually reduced in December (Supplement Figure 1).

\section{$\mathrm{RF}(\mathrm{IU} / \mathrm{mL})$ \\ $\mathrm{E} 2(\mathrm{pg} / \mathrm{mL})$}

FSH $(\mathrm{mIU} / \mathrm{mL}$

$\operatorname{CRP}(\mathrm{mg} / \mathrm{dL})$
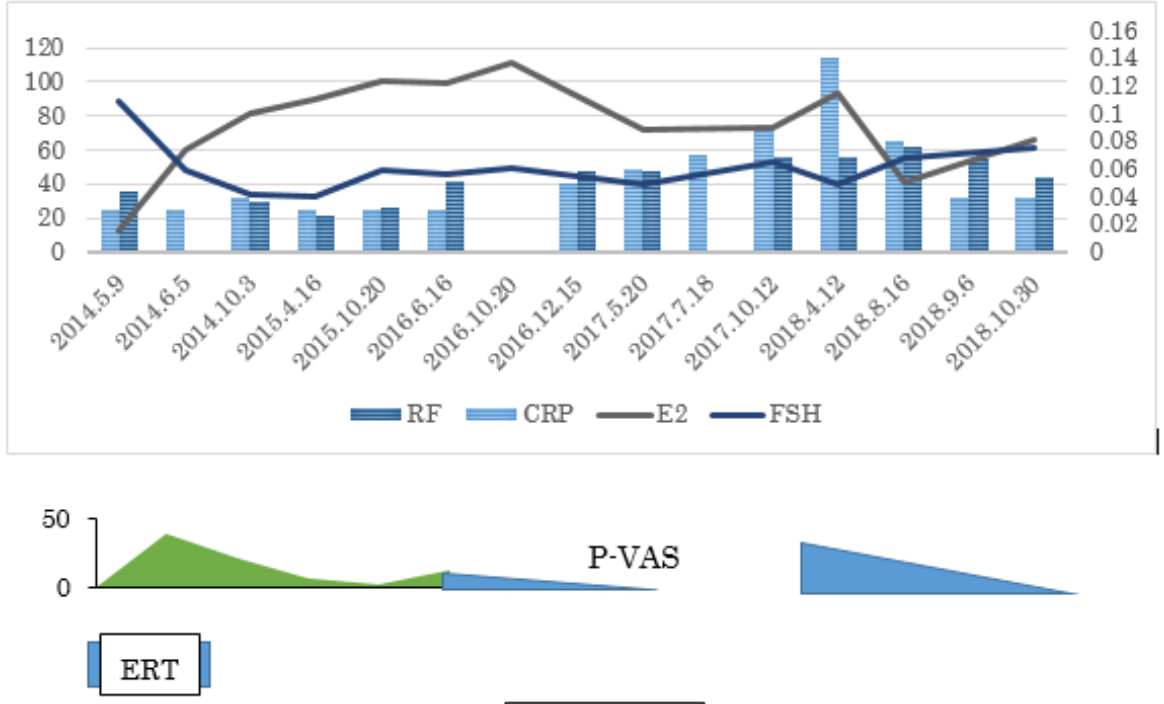

EPRT cyclic

Supplement Figure 1: Clinical and laboratory findings of Case 1.

HRT started immediately on the first visit and her joint pain was reduced to $39 \%$ at one month later, and $7 \%$ at 10 months later. She was diagnosed as having postmenopausal arthralgia for the past 4 years. However, she newly complained of left wrist joint pains in August in 2018 and $X$ ray showed bone erosion associated with radiocarpal joint space narrow and received 8mg weekly. Her pain was gradually reduced in December.

Case 2 was born in 1972. She received Kaufmann therapy from 2010 to 2014, but it ended treatment because of no improvement, and she was diagnosed as menopause. She started feeling uncomfortable at the fingers joints and increasing the number of hair loss. She got tired and hard to get up in 2015. She visited at orthopaedic clinic because of evaluation of increasing joint pain in Autumn 2016. Her blood examination revealed CRP 0.03, RF 5, Anti-CCP 3, MMP3 36.8 and ANA 40 homogenous in March 2018. However, she was diagnosed as a RA because of a new erosion and recommended to receive biologic DMARD by Rheumatologist. She moved my clinic in August 2018. HRT started immediately, her joint pains was reduced to $50 \%$ one month later and $20 \%$ in December 2018. (Supplement Figure 2). Her ANA going up to 160 with homogenous staining was proved anti-DFS70 Ab 80.0 Index positive.

These cases were initially diagnosed as having RA and received MTX at previous rheumatologists, but they wondered whether these joint pains were related to menopause or not. There are so many women presenting peripheral joint pains, sometimes with big joints. We explored to evaluate UA at peri-and post-menopausal stage. 


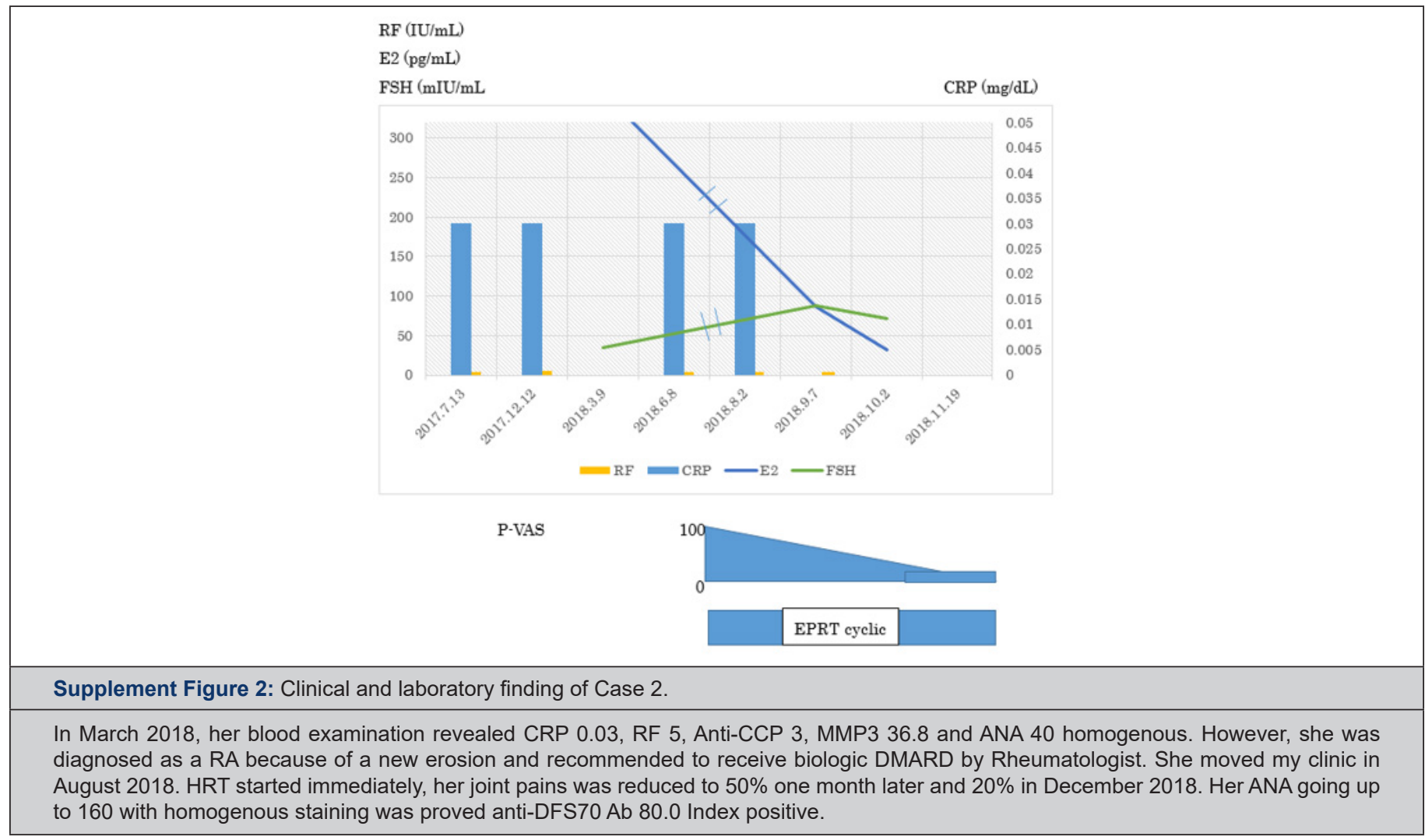

\section{Methods}

\section{Setting and patients}

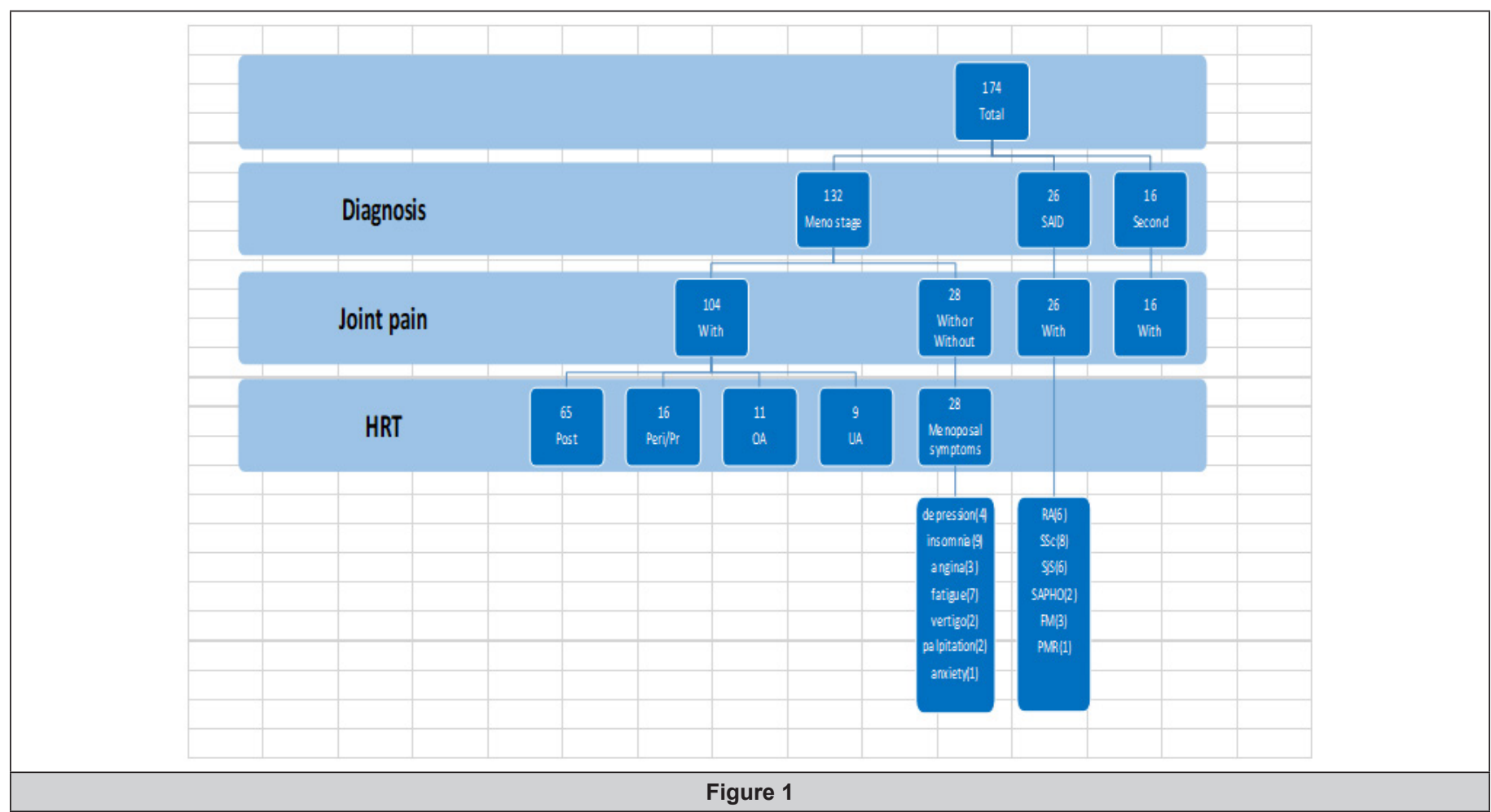

174 female ethnically Japanese patients aged between 36 and 77 who visited a single women's outpatient rheumatology clinic between January and September were screened (Figure 1). Inclusion criteria were female sex, menopausal symptoms, and undifferentiated arthritis, defined as tenderness in two or more joints and pain on joint motion, with or without morning stiffness. Patients meeting respective criteria for other pathology, such as rheumatoid arthritis (defined as ACR/EULAR criteria $\geqq 6$ points), 
Sjogren's syndrome (SjS) [8], systemic lupus erythematosus (SLE) [9], or systemic scleroderma (SSc) [10], were excluded. Patients with joint erosions on x-ray. Twenty-seven declined further involvement in the study. The remaining 82 were provided with a detailed explanation of the scope and purpose of the study and gave their written and/or oral consent prior to enrolment. This study was approved by the Ethics Board of the Society of Healthcare for Menopause and Aging in Yokohama, Japan.

\section{Criteria for menopausal status}

Criteria used to define postmenopausal state were one year since last menstruation, serum estradiol (E2) level of less than 20pg/ml (Chemilumi ACS-Estradiol-6, Siemens Healthcare Diagnostics), and follicle stimulating hormone (FSH) level greater than $30 \mathrm{mIU} / \mathrm{ml}$ (Chemilumi ACS-FSH, Siemens Healthcare Diagnostics). Perimenopausal state was defined as age greater than 45 years, and irregular menstruation.

\section{HRT Protocol}

HRT was administered as per the Japanese Society of Menopause and Health Care guidelines [11]. In brief, postmenopausal women with past history of hysterectomy received estrogen replacement therapy using $17 \beta$ estradiol patch $(0.72 \mathrm{mg}) / 2$ days. Postmenopausal women without hysterectomy aged under 55 received estrogen ( $17 \beta$ estradiol plaster) and progesterone (dydrogesterone 10mg) replacement therapy (EPT) with cyclic treatment, and those in this group over 55 received the same with continuous EPT. Eight women aged over 60 in this group received E3 at 1 to $2 \mathrm{mg}$ per day, plus progesterone (dydrogesterone $5 \mathrm{mg}$ ) for 12 days every 6 months. Perimenopausal women received Tocopherol Nicotinate $600 \mathrm{mg} /$ day.

\section{Study end-point criteria}

All patients were assessed 2 months post treatment commencement. Patients with small joint involvement only and greater than $30 \%$ reduction in joint pain as defined by patient (p)-VAS scores were considered to have responded to treatment. Patients with large joint involvement (knee or elbow) were assessed at 6 months for fulfilment of the same criteria. All patients were considered to have a baseline p-VAS score of 100 at the start of the trial. Those who responded to treatment were reclassified as peri-, or postmenopausal arthralgia, respective to their menopausal status.

\section{Results}

\section{Screening and enrolment}

Clinical and serological results were used to make tentative diagnoses following two visits. Of the original 174 women screened, 6 were diagnosed with RA, 4 with depression with arthralgia, 6 with SjS, 12 with OA, 8 with localized SSc, 3 with fibromyalgia (FM), and 1 with polymyalgia rheumatica. 83 women remained classified as undifferentiated arthritis and were enrolled in the study, of whom were, 15 were perimenopausal, and 68 were postmenopausal.

\section{Efficacy of HRT in postmenopausal UA}
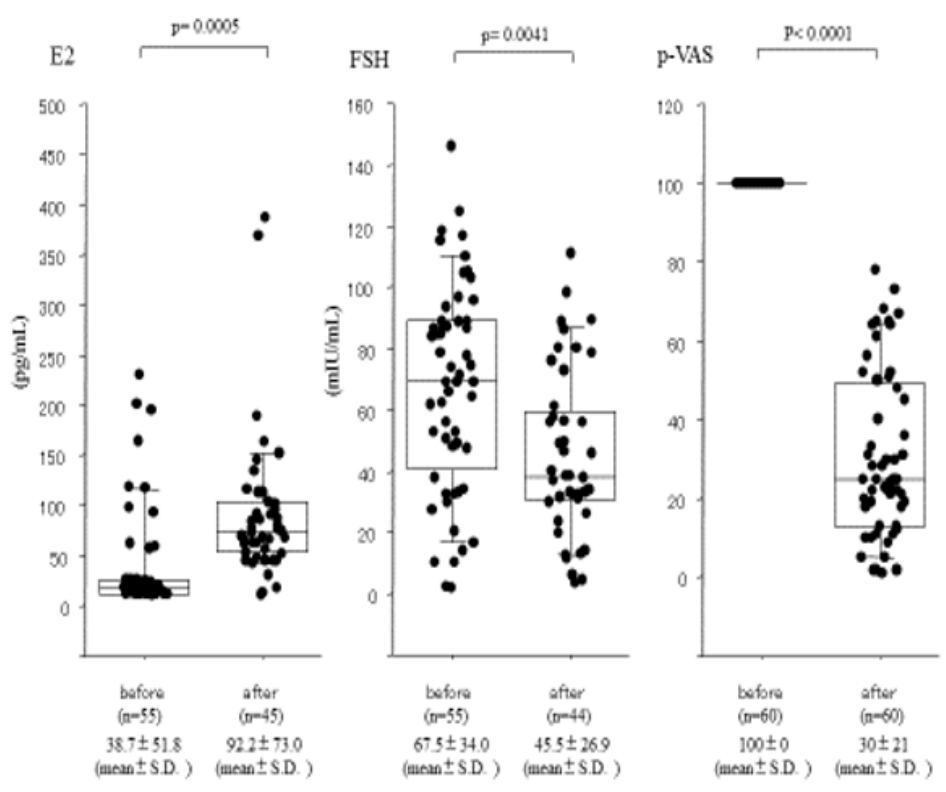

Figure 2: Efficacy of HRT for postmenopausal 68 women with UA.

Comparison of E2, FSH and p-VAS levels before and after treatment with HRT for 2 to 6 months.

68 women with UA were classified as postmenopausal and received HRT. Of these, $30 \%$ or greater reduction in p-VAS, and were therefore diagnosed with postmenopausal arthralgia following treatment. Eight women over 60-year-old treated with E3 2mg/day were excluded because of lack elevation of E2. Therefore, Pre- and post-treatment E2, FSH, and p-VAS were obtained in 60 women. 
Baseline levels of E2 and FSH in this group were $38.7 \pm 51.8 \mathrm{pg} / \mathrm{ml}$ and $67.5 \pm 34.0 \mathrm{mIU} / \mathrm{ml}$, respectively, consistent with postmenopause. Following administration of HRT for two to six months, E2 and FSH levels were $92.2 \pm 73.0(p<0.0005)$ and $45.5 \pm 26.9(p<0.0041)$, respectively, indicating adequate recovery of hormone levels. p-VAS scores improved significantly from $100 \pm 0$ to $30 \pm 21(\mathrm{p}<0.0001)$ (Figure 2) and most patients reported resolution of morning stiffness, within two weeks.

\section{Efficacy of Tocopherol $\mathrm{N}$ in perimenopausal UA}
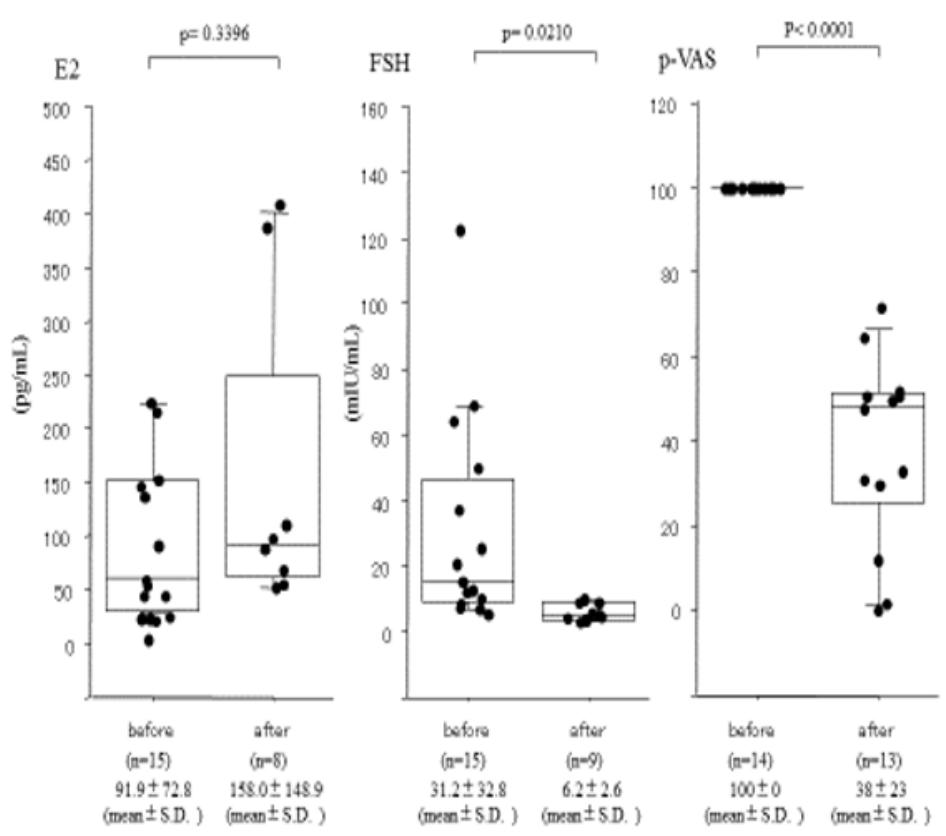

Figure 3: Efficacy of Tocopherol Nicotinate $600 \mathrm{mg} /$ day for perimenopausal women with UA. Comparison of E2, FSH and p-VAS levels before and after treatment with Tocopherol Nicotinate $600 \mathrm{mg} /$ day for 2 to 6 months. respectively. $p$-VAS improved from $100 \pm 0$ to $31 \pm 3(p=0.0001)$.

Fifteen women with UA were classified as perimenopausal and were treated with tocopherol N. Of these, Pre-and post-treatment E2, FSH and p-VAS were obtained in 15 patients. Baseline E2 and FSH levels were $91.9 \pm 72.8 \mathrm{pg} / \mathrm{ml}$ and $31.2 \pm 32.8 \mathrm{mIU} / \mathrm{ml}$, respectively. These results were consistent with perimenopause. Following administration of Tocopherol $\mathrm{N}$ for two to six months, E2 and FSH levels were $158.0 \pm 148.9(p=0.0016)$ and $6.2 \pm 2.6(p<0.05)$, respectively, indicating adequate treatment. p-VAS improved greatly from $100 \pm 0$ to $31 \pm 3(\mathrm{p}=0.0001)$. (Figure 3 ), indicating significant symptomatic relief in this group. However, these data were so small and E2, FSH and pVAS were not completed simultaneously that further study is needed.

\section{Discussion}

There has been little study of the relationship between arthralgia and menopause. However, as estrogenic is known to act as an inhibitor of the inflammatory response [12], it is worth considering whether serum estrogenic reduction during the peri- and postmenopausal period plays a role in promoting joint inflammation in women during this period. It has previously been shown that relative reduction in estrogenic levels induced transient activation of T- and B-lymphocytes, as well as production of inflammatory cytokines including IL-6 and TNF $\alpha$ [13] that are hallmarks of the chronic inflammatory response [14]. Clinically, mid-aged women having severe myalgia /arthralgia are associated with higher incidence of insomnia/depression, in contrast to having slight myalgia/arthralgia. These symptoms are closely related and may be induced by estrogen deficiency [15].

ACR/EULAR criteria 2010 for early stage are very useful for finding new patients with RA, however, this criterion was not concerned with menopausal arthralgia well. The swelling of joint pain is not required and tenderness or pain on motion is accepted for joint problems, leading to misdiagnosis of RA. Recently, $4780 \mathrm{RA}$ patients following 3 years were reassessed and among them, 39.3\% were misdiagnosed and 50.1\% RA [7]. Misdiagnosed RA consisted of $50.1 \%$ with osteoarthritis (OA), 39.3\% with unknown and only $10.6 \%$ with autoimmune rheumatic disease [16]. OA is also related to occur at menopausal stage but is rarely found in male around 50 . OA may be mainly associated with estrogen deficiency. If so, $89.4 \%$ of misdiagnosed RA is mainly caused by estrogen deficiency.

In Japan, HRT is not used widely and limited in women's health clinic, so only $2 \%$ of menopausal women received HRT, in contrast to $20 \sim 50 \%$ in USA Europe and Australia. Therefore, many menopausal women complained of peripheral joint pain, sometimes including big joints, visited the orthopaedic clinic or rheumatologist in the hospital and they wondered whether they had RA or not. When doctors could not make a final diagnosis, 
menopausal women will have to go around a doctors shopping. In such situation, HRT will be recommended. If it works, they have a postmenopausal arthralgia/arthritis caused by estrogen deficiency [17], because estrogen supplementation mostly relieved joint pain in postmenopausal women.

Clinical association between UA and progression to RA has been reported frequently. Van der Helm-van Mil et al. [18] reported that approximately $40 \%$ of cases of UA progressed to RA within one year, $20 \%$ to other diseases, and the remaining $40 \%$ exhibited remission. Furthermore, Kaneko Y et al. [19], in their assessment of the sensitivity of the 2010 RA Classification Criteria found sensitivity reduced to $15.8 \%$ where patients were rheumatoid factor (RF) and anti-CCP antibody negative, in contrast with a baseline sensitivity of $73.5 \%$.

As for anti-DFS $70 \mathrm{Ab}$, two demonstrated cases shown above had high titer of ant-DFS $70 \mathrm{Ab}$. After they received HRT, joint pains were reduced to $10 \sim 24 \%$ from maximum $100 \%$, respectively. However, the first case having low titer of anti-CCP Ab developed RA, although four year's receiving HRT. The existence of anti-DFS $70 \mathrm{Ab}$ was not protective for ongoing to RA in this case, under the treatment of HRT [20].

The pathogenesis of RA is complex, and much of this process remains to be determined. This study provides preliminary findings which indicate that estrogen may be of vital importance in the onset of this disease, and supplementation may provide significant symptomatic relief in peri- and postmenopausal women with UA. Further studies are needed to confirm these findings and explore possible use of HRT or Pills for the prevention of progression to RA in these patient groups [21,22].

\section{Acknowledgement}

The authors thank Dr Sousei Kou of Enoshima Women's Clinic for discussion and encouragement. Earlier progress of this study was reported at the $60^{\text {th }}$ Japanese College of Rheumatology conference in 2016.4.28. (Yokohama, Japan) and the $30^{\text {th }}$ Japanese Women's Health and Aging conference in 2015.11.9.

\section{References}

1. Nagamine R, Maeda T, Shuto T, Nakashima Y, Hirata G, et al. (2001) Menopausal syndrome in female patients with rheumatoid arthritis. Mod Rheumatol 11(3): 230-233.

2. Mitchell ES, Woods NF (2010) Pain symptoms during the menopausal transition and early postmenopause. Climacteric 13(5): 467-478.

3. Yasui $T$, Uemura $H$, Irahara $M$, Arai M, Kojimahara N, et al. (2008) Associations of endogenous sex hormones and sex hormone-binding globulin with lipid profiles in aged Japanese men and women. Clin Chim Acta 398(1-2): 43-47.

4. Murphy AJ, Guyre PM, Pioli PA (2010) Estradiol suppresses NF-kappa $B$ activation through coordinated regulation of let-7a and miR-125b in primary human macrophages. J Immunol 184(9): 5029-5037.

5. Giraud SN, Caron CM, Pham-Dinh D, Kitabgi P, Nicot AB (2010) Estradiol inhibits ongoing autoimmune neuroinflammation and NFkappa B-dependent CCL2 expression in reactive astrocytes. PNAS 107(18): 8416-8421.
6. Van der Linden MP, Knevel R, Huizinga TW, Van der Helm-van Mil AH (2011) Classification of rheumatoid arthritis: comparison of the 1987 American College of Rheumatology criteria and the 2010 American College of Rheumatology/European League Against Rheumatism criteria. Arthritis Rheum 63(1): 37-42.

7. D Gomez, G Saavedra-Martinez, L Villarreal, P J Bello-Gualtero, V Giraldo, et al. (2015) Misdiagnosis of Rheumatoid Arthritis - The Photography. Ann Rheum Dis 74 (2): 689.

8. Fujibayashi T, Sugai S, Miyasaka N, Hayashi Y, Tubota K (2004) Revised Japanese criteria for the Sjogren's syndrome (1999): availability and validity. Mod Rheumatology 14(6): 425-434.

9. Tan EM, Cohen AS, Fries JF, Masi AT, McShane DJ, et al. (1982) The 1982 revised criteria for the classification of systemic lupus erythematosus. Arthritis Rheum 25(11): 1271-1277.

10. Le Roy EC, Black C, Fleischmajer R, Jablonska S, Krieg T, et al. (1988) Scleroderma (systemic scleroderma): classification, subsets and pathogenesis. J Rheumatol 15(2): 202-205.

11. Suka M, Taniuchi A, Kudo Y, Sato S, Yoshida K, et al. (2010) Self-assessed health and menopausal symptoms among 50 -year-old Japanese women: cross-sectional surveys in Northern Kawasaki in 1998 and 2008. Menopause17(1): 166-173.

12. The Americans College of Obstetrics and Gynecologists (2015) Women's Health Care Physicians. Patient education Fact Sheet. PFS003 Hormone Therapy.

13. Cheng CC, Chen YH, Chang WL, Yang SP, Chang DM, et al. (2010) Phytoestrogen bavachin mediates anti-inflammation targeting Ikappa B kinase-I kappaB alpha-NF-kappaB signaling pathway in chondrocytes in vitro. Eur J Pharmacol 636(1-3): 181-88.

14. Pacifici R, Brown C, Puscheck E, Friedrich E, Slatopolsky E, et al. (1991) Effect of surgical menopause and estrogen replacement on cytokine release from human blood mononuclear cells. Proc Natl Acad Sci USA 88(12): 5134-5138.

15. Masakazu Terauchi, Asuka Hirose, Mihoko Akiyoshi, Kiyoko Kato, Naoyuki Miyasaka (2017) Muscle and joint pains in middle-aged women are associated with insomnia and low grip strength. A cross-sectional study, Journal of Psychosomatic Obstetrics \& Gynaecology 100: 136.

16. Vural P, Akgul C, Canbaz M (2006) Effects of hormone replacement therapy on plasma pro-inflammatory and anti-inflammatory cytokines and some bone turnover markers in postmenopausal women. Pharmacol Res 54(4): 298-302.

17. Miyachi K, Ihara A, Sasse B (2018) Does hormone replacement therapy prevent undifferentiated arthritis progressing to rheumatoid arthritis. Eular Amsterdam THU0695 poster 77(2).

18. Van der Helm-van Mil AH, le Cessie S, van Dongen H, Breedveld FC, et al. (2007) A prediction rule for disease outcome in patients with recentonset undifferentiated arthritis: how to guide individual treatment decisions. Arthritis Rheum 56(12): 433-40.

19. Kaneko Y, Kuwana M, Kameda H, Takeuchi T (2011) Sensitivity and Specificity of 2010 Rheumatoid Arthritis Classification Criteria. Rheumatology (Oxford) 50(7): 1268-1274.

20. RL Ochs, Michael M, A Basu, L Rios-Colon, TW Sanchez, et al. (2016) The significance of autoantibodies to DFS70/LEDGFp75 in health and disease: integrating basic science with clinical understanding. Clin Exp Med 16: 273-293

21. Hazes JM, Dijkmans BC, Vandenbroucke JP, de Vries RR, Cats A (1990) Reduction of the risk of rheumatoid arthritis among women who take oral contraceptives. Arthritis Rheum 33(2): 173-179.

22. Orellana C, Saevarsdottir S, Klareskog L, Karlson EW, Alfredsson L, et al. (2015) Postmenopausal hormone therapy and the risk of rheumatoid arthritis: results from the Swedish EIRA population-based case-control study. Eur J Epidemiology 30(5): 449-457. 